\title{
Self-Compassion and Breast Cancer in 23 Cancer Respondents: Is the Way You Relate to Yourself a Factor in Disease Onset and Progress?
}

\author{
Karen G. Kearney, Richard E. Hicks* \\ School of Psychology, Faculty of Society and Design, Bond University, Gold Coast, Australia \\ Email: *rhicks@bond.edu.au
}

How to cite this paper: Kearney, K. G., \& Hicks, R. E. (2017). Self-Compassion and Breast Cancer in 23 Cancer Respondents: Is the Way You Relate to Yourself a Factor in Disease Onset and Progress? Psychology, 8, 14-26.

http://dx.doi.org/10.4236/psych.2017.81002

Received: November 15, 2016

Accepted: December 26, 2016

Published: December 30, 2016

Copyright $\odot 2017$ by authors and Scientific Research Publishing Inc. This work is licensed under the Creative Commons Attribution International License (CC BY 4.0).

http://creativecommons.org/licenses/by/4.0/ (c) (i) Open Access

\begin{abstract}
Breast Cancer has been extensively studied. However, there is limited knowledge about how psychological factors such as levels of self-compassion may be related to disease onset. This study examined whether higher levels of selfcompassion may be associated with a delayed onset of cancer and its progression and lower hyper-arousal scores in Australian women with breast cancer, using retrospective recollections as part of the study design. Participants from Australian cancer support groups were invited to complete an online survey including demographic and cancer history information, a hyper-arousal scale and a self-compassion scale (self-report scales). After data screening 23 of the 31 responding participants were included in the study. Correlation and regression analyses were used to analyse the relationships. Higher levels of selfcompassion were related to later onset of breast cancer and to lower hyperarousal scores. The findings suggest that self-compassion may be a protective factor in relation to hyper-arousal and early onset and progression of disease. The implication, subject to more extended research, is that counsellors, therapists and physicians may reduce the personal and health costs of those with breast cancer by helping them become more aware of and better users of effective self-compassion strategies.
\end{abstract}

\section{Keywords}

Health Psychology, Breast Cancer, Self-Compassion, Hyper-Arousal, Therapy

\section{Introduction}

Breast cancer is one of a number of common, painful, life-threatening illnesses. In general terms much is known about genetic and non-genetic influences in re- 
lation to disease onset and progression, including the relationship between psychosocial stress, neuroendocrine-immune changes and increased risk of cancer (e.g., Sephton \& Spiegel, 2003). We do know that emotions affect outcomes in breast cancer via mood states such as depression (Kanani, Davies, Hanchett, \& Jack, 2016) and that in general health terms studies in related fields such as prostate cancer (Sharp, O'Leary, Kinnear, Gavin, \& Drummond, 2015) have recommended interventions aimed at decreasing depression, anxiety and stress levels in cancer patients. However, little attention has been given to emotion regulation strategies such as self-compassion as an intervention that may influence how individuals with cancer manage stress and the subsequent physiological hyper-arousal responses that can affect immune system functioning.

Our study examined in a sample of those with breast cancer, the relationships between cancer onset and attitudes held towards oneself (self-compassion). Selfcompassion is a self-regulation strategy that calms the emotions (an internal strategy) and enables individuals to deal more positively with the outside environment (external to themselves and their thinking). Background research on self-compassion is discussed next and then we turn to breast cancer (onset and progress of the disease in relation to hyper-arousal or stress issues), before linking self-compassion, hyper-arousal and breast cancer and developing hypotheses.

\subsection{Self-Compassion}

Self-compassion involves an emotion regulation strategy in which we accept ourselves and our weaknesses and where we do not avoid or suppress negative experiences (Neff, 2003a). The opposite of self-compassion (not being selfcompassionate) occurs when we use avoidance strategies. This raises internal stress responses which are more likely, then, to engage chemicals such as cortisol (Gilbert, 2002). Self-compassion strategies involve kindness and understanding toward the self (Neff, 2003a; Neff et al., 2007) in the midst of an awareness of distressing feelings.

Self-compassion involves recognising that suffering and disappointment are part of being human and that people are worthy of kindness and compassionate care (Neff, 2003a). When compassion is applied to the self, we become open to our own suffering and pain and allow an accepting kindness toward our inadequacies. Often people are less kind to themselves and engage in strategies that involve over emphasis on and over-identification with the situation, or they disconnect themselves from the collective human experience of suffering, feeling isolated and alone. Self-compassion allows an individual to view the experience as though from an outside perspective, a mindful and more balanced view, lessening the impact of the negative experience (Neff, 2003a).

\subsubsection{Self-Compassion Benefits: Biopsychosocial Implications}

Studies that have investigated compassion and stress related immune responses have found positive outcomes for those utilising this concept. For example, Pace 
and colleagues found that people who engaged in higher levels of compassion focused meditation scored lower on physiological immune related stress responses such as cortisol levels and on psychological measures of distress; suggesting that stress induced immune and behavioural responses may be moderated by compassion focused meditations (Pace et al., 2009). A study on an autoimmune disease (scleroderma) and self-compassion found that lower levels of hyper-arousal (a response that can negatively impact on the immune system) were related to greater levels of self-compassion and a later onset of disease (Kearney \& Hicks, 2016). In the study by Wren et al. (2011), the effects of selfcompassion lead to better management of persistent musculoskeletal pain. A study exploring self-compassion in women experiencing body changes resulting from breast cancer treatment found that self-compassion was a protective factor for reducing psychological distress (Przezdziecki et al., 2013). The conclusion is therefore that self-compassion strategies may reduce physiological arousal and the subsequent negative immune responses (Pace et al., 2009; Przezdziecki et al., 2013). Self-compassion may therefore be an important strategy in the treatment of breast cancer. There are however, few studies that have explored the breastcancer and self-compassion interactions in detail. Our examination of the research on self-compassion in relation to health problems suggested that strategies high in self-compassion may produce positive physical and psychological health outcomes for those with breast cancer.

\subsubsection{Summary: Why Does Self-Compassion Seem to Work?}

This strategy provides a more positive emotion regulatory approach, as it is not based on an evaluative process (Neff, 2003a) involving the fight and flight/threat system (Gilbert, 2007), but on kindness and understanding toward the self (Neff, 2003a; Neff et al., 2007). Self-compassion provides a solution for threat processing difficulties, and is negatively associated with depression, anxiety, self-criticism and thought suppression and positively associated with life satisfaction (e.g., Gilbert 2007; Neff, 2003a; Neff et al., 2007), stress reduction (Sharpiro, Astin, Bishop, \& Cordova, 2005) and lower cortisol levels (Pace et al., 2009). Self-compassion is significantly related to adaptive functioning and positive health outcomes (Neff et al., 2007).

\subsubsection{Self-Compassion in Relation to Hyper-Arousal and Age of Onset of Cancer}

Self-compassion was hypothesised to act as a protective factor for immune functioning in that higher levels of self-compassion would be related to lower physiological stress experiences (hyper-arousal) and to more positive health outcomes (that is, that individuals emphasising this emotion regulation strategy would have experienced a later onset of breast cancer than those not emphasising the strategy). We use the past tense here to refer to age of onset of cancer as our study is not following cohorts through a life period but instead requesting retrospective comments. These are subject to error as are all self-report measures and studies; however, in the time and funding constraints we faced, significant 
relationships could be identified and implications drawn for future more extensive studies.

\subsection{Breast Cancer}

Cancer is the main cause of illness in Australia and in 2012 it was anticipated that approximately 120000 Australians would receive a cancer diagnosis. Breast cancer is one of the most common forms of cancer that develops in women; it also occurs in men; however, it is much rarer (1: 100). In 2010, breast cancer accounted for $15 \%$ of cancer deaths in women and was the second highest cause of cancer-related deaths in women (2840 women and $24 \mathrm{men}$ ). The incidence of breast cancer is rising and it is predicted that by 2020 over 17,000 new cases will be diagnosed in Australian women (Australian Institute of Health and Welfare \& Australasian Association of Cancer Registries, 2012).

\subsubsection{Breast Cancer and Stress}

Research also suggests there are associations between neuroendocrine-immune changes, psychosocial stress and increased risk of cancer (Sephton \& Spiegel, 2003). A number of studies have reported associations between stress and trauma in individuals diagnosed with cancer. A study conducted into adverse childhood experiences found a possible relationship between multiple adverse childhood experiences reported by $62 \%$ of participants. Cancer diagnosis was reported by $10 \%$ of participants (Brown, Thacker, \& Cohen, 2013). A breast cancer comparison study in relation to disease-free intervals was conducted by Palesh and colleagues (2007) between female participants who experienced stress/traumatic event/s and females reporting no stress/trauma event. Results demonstrated that significantly longer disease-free intervals occurred amongst participants with no stress/trauma, while those reporting stress/trauma, experienced the shortest disease-free interval. Results indicated a link between stressful or traumatic experiences and more rapid breast cancer progression and the possibility that stressful or trauma events may reduce the capacity of the individual's immune system to resist tumour growth (Palesh et al., 2007).

Our study aimed to identify at least the initial relationships among self-compassion attitudes and strategies used, and the effects on hyper-arousal in our sample of Australian women with breast cancer.

\subsubsection{Breast Cancer and Stress Experience, and Hypotheses on Hyper-Arousal}

There is a need to find strategies to manage exposure to stress/trauma events that reduce the impact on the immune system. Self-compassion may through the reduction of negative emotion, provide opportunities to manage physiological aspects of the stress response (such as hyper-arousal) and the subsequent immune reaction that may lead to disease onset and progression. We therefore examined these aspects in our hypotheses.

\subsection{Hyper-Arousal and the Immune System}

Hyper-arousal is a physiological response that involves the fight and flight sys- 
tem (e.g., Every \& Lating, 2002) and is associated with psychological conditions and dysregulation of the immune system (Schore, 1994). Hyper-arousal is described as physical or emotional tension produced by hormones during the fight-or-flight response. The intensity of this response is generally dependent on an individual's response to a stressor (e.g., Every \& Lating, 2002; Selye, 1976) and ability to adapt to repeated exposure to unexpected stimuli which may produce excessive arousal reactions and increased cortisol levels (Hammad, Barsky, \& Regestein, 2001). Therefore, we hypothesised that if the responses can be affected positively, that there would be benefits in delaying onset and progress of cancer: we examined self-compassion as a strategy that would yield more positive personal responses and be more effective in those with cancer.

\section{Impacts of These Arousal Hormones}

High levels of the stress hormone cortisol can have negative effects on the immune system, while epinephrine and norepinephrine affect the sympathetic nervous system (SNS: Rice, 1999). Norepinephrine increases fight and flight reactions and increases hyper-vigilance to threat (Every \& Lating, 2002). The release of dopamine into the amygdale (which strives to deal with emotions) creates a conditioned fear response as a result of an over-reactive amygdala intensifying the arousal-related fear response (Every \& Lating, 2002).

We concluded that as hyper-arousal is involved in the stress response and the immune system (e.g., Every \& Lating, 2002), it may also be a condition associated with individuals diagnosed with breast cancer.

\subsection{Self-Compassion, Hyper-Arousal and Disease Onset}

Based on the available literature related to self-compassion, this study explored whether strategies high in self-compassion protect an individual from elevated negative arousal through the reduction of negative emotion. Self-compassion is therefore hypothesised to be a strategy that may act as an emotional and physiological regulator that lessens the impact on the immune system and therefore disease onset, through the reduction of hyper-arousal. It is therefore postulated that individuals reporting a later onset of breast cancer and lower experiences of hyper-arousal would have utilised strategies higher in self-compassion than those reporting earlier disease onset and elevated levels of hyper-arousal.

\subsection{Hypotheses: Self-Compassion, Hyper-Arousal and Age Diagnosed with Breast Cancer}

Essentially our hypotheses were straightforward: that greater Self-compassion would be associated with lower Hyper-arousal and illness onset at an older age, whereas lower Self-compassion would be related to greater Hyper-arousal and a diagnosis of Breast Cancer at a younger age. We used Pearson Product Moment correlations to examine these hypotheses. We then combined these variables in regression analyses that (1) identified if hyper-arousal and age of onset were related to (predicted) self-compassion levels, and (2) identified if reported selfcompassion levels predicted hyper-arousal and separately age of onset-we were 
examining relationships and whether hyper-arousal and age of onset would be related to self-compassion strategies. We hypothesised the relationships would be strong and therefore hoped to conclude that self-compassion strategies should be used in clinical practice to help those with breast cancer (though this conclusion would need further follow-up research using randomised control design-experimental treatment comparison-approaches to confirm the conclusion). Please see Figure 1 below for a visual representation of the theory and hypothesis.

\section{Method}

\subsection{Procedure}

Breast cancer organisations and support groups reaching an international population were contacted to determine interest in promoting this study to members diagnosed with breast cancer. In the end we obtained a sample of 23 fully usable forms. A small response rate, as discussed subsequently, is not unusual in such research. Several organisations reported some concern about exploring the psychological experiences of their members diagnosed with breast cancer and access of members to information about the study was denied, thus reducing the opportunities to develop a larger sample in the time allowed. The Breast Cancer Care Organisation from Western Australia and the West End Advanced Breast Cancer Group in Queensland gave access. Accordingly, information explaining the purpose of the study was placed on the Western Australian Breast Cancer Care's websites notice board and included a link to the online survey (hosted on Bond University's research website). Information about the study was also displayed on a poster on the notice board at the Advanced Breast Cancer Support Group in Brisbane with similar information on web access to the survey. A notice was also posted on the Research Participation Notice Board of the School of Psychology. A written explanatory letter containing information about the purpose, procedure, where questions/complaints could be directed, risks and benefits of the research project and anonymity of participants was attached as a cover

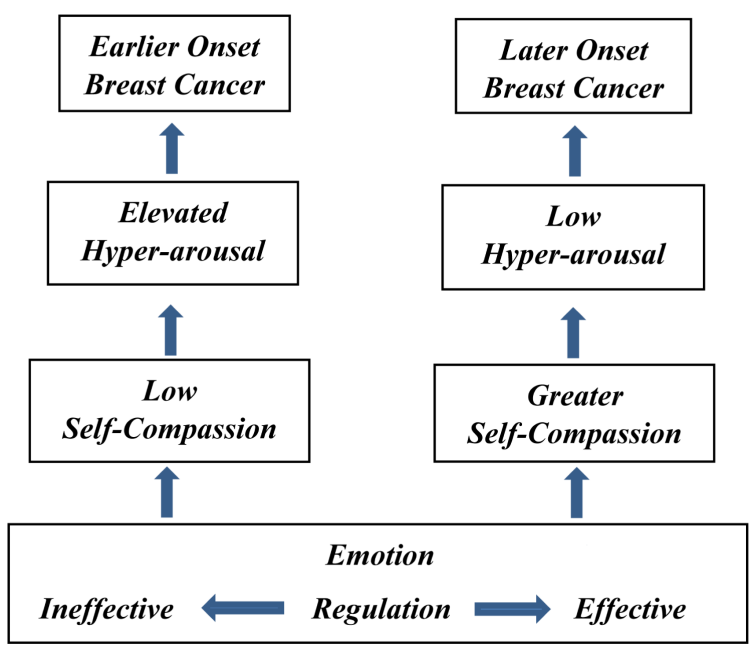

Figure 1. Model of theoretical framework and hypothesis. 
page to the questionnaire for participants to view prior to completing the survey. Indication that participants could withdraw at any time for any reason was also given. Completion of the survey required approximately 30 minutes of the participant's time. This project was approved by the University's Human Research Ethics Committee.

\subsection{Participants}

Male and female adults aged 18 years and over who had been diagnosed with breast cancer, were invited to participate in this research project. Thirty-one individuals diagnosed with breast cancer from breast cancer organisations and staff and students from the University commenced the online survey, though just 23 completed all aspects reported in the current paper.

\subsection{Measures}

Breast cancer related information such as age diagnosed with breast cancer and category, stage and type of breast cancer (such as ductal carcinoma in situ, early breast cancer, and inflammatory breast cancer) were included in the survey.

\subsubsection{Self-Compassion: Self Compassion Scale}

The Self-Compassion Scale (Neff, 2003b) has been widely used to measure SelfCompassion. This scale is a 26 item, self-report, emotion regulation measure, that employs a 5 point Likert scale (e.g., $1=$ almost never to $5=$ almost always) containing three components. Self-kindness/Self-judgment (being kind and understanding toward oneself rather than judgmental or critical: 10 items); Common humanity/Isolation (viewing one's negative experiences as a normal part of the human condition rather than experiencing suffering in isolation: 8 items); and Mindful acceptance/Over-identifying (being open to and accepting of one's situation rather than over-identifying with painful thoughts and feelings: 8 items). Examples of items include, "When things are going badly for me, I see the difficulties as part of life that everyone goes through." "I try to be loving towards myself, when I'm feeling emotional pain." "When I feel inadequate in some way, I try to remind myself that feelings of inadequacy are shared by most people." This scale has good psychometric properties (construct, content, convergent and discriminant validity, test-retest reliability) and is a valid theoretical measure of self-compassion (Neff, 2003b). Self-compassion may be an adaptive process that increases psychological resilience and well-being (Neff, 2003b).

\subsubsection{Arousal: The Hyper-Arousal Scale}

The Hyper-arousal Scale (Hammad, Barsky, \& Regestein, 2001) is a 26 item selfreport that measures tendencies to introspect about feelings and respond intensely to unexpected stimuli and other behaviours that involve cortisol arousal. The scale contains questions involving "Bright lights \& crowds and thinking a lot about feelings." This scale has good psychometric properties (predictive and construct: Hammond, Barsky, \& Regestein, 2001). Responses to each item on the Hyper-arousal Scale are graded from 0 to 3 respectively where $0=$ not at all, $1=$ 
a little, 2 = quite a bit and 3 = extremely. Hyper-arousal scale scores correlate with several EEG measures of arousal including frequency of spectral and evoked potential measures. Hyper-arousal scores signify increased general cerebral responsiveness but decreased selective attention, indicating openness to stimuli and difficulty distinguishing between physiologically harmless and threatening sensations. This situation may create an information overload and result in difficulty adapting to recurring stimuli. A decrease in selective attention may create ambiguity as to the meaning attributed to the perceived experience resulting in the development of an adversity management system.

\section{Results}

\subsection{Analyses}

Analysis was performed using SPSS version 23. Results demonstrated the variables to be sound, with good to satisfactory internal consistency psychometrics (Cronbach's alpha coefficients were above .80 for both the Self-Compassion and the Hyper-arousal scales). Descriptive statistics, means and standard deviations and frequencies for demographic information such as age, and health (including age diagnosed with breast cancer) were extracted. Of the total of 31 subjects 28 were female and one male; two participants failed to give their age or gender.

Age

Frequencies for current age were reported at between 37 and 76 years with a mean age of 54; for age diagnosed with breast cancer, age for first diagnosed with breast cancer was between 36 and 69 years, mean age was 50 .

Missing data was evident, the pattern appeared random for all completed scales. A large number of participants had completed demographic, health and breast cancer information. However, approximately 30\% did not complete the psychological scales. Thirty one individuals participated in the study; with the exclusion of cases to meet assumptions and hypothesis criteria a total of 23 participants remained. Low participation rates are evident in cancer and related studies. Research suggests that the number of breast cancer participants prepared to engage in research studies is between $1.2 \%$ and $11 \%$ of available participants (Habersack \& Luschin, 2013). For example, Habersack and Luschin's (2013) study compared 12 breast cancer participants with 10 non-breast cancer participants on psychosocial variables. It seems that the pain and stress of involvement in studies such as we are attempting affects people's willingness and also their ability to participate and hence lower numbers respond in such studies and fewer complete them. However, we can draw valuable insights from small numbers as we demonstrate from the findings and implications of our study.

\subsection{Examining the Hypotheses: Bivariate Correlation Coefficients}

Pearson's bivariate correlations were accepted as appropriate measures of relationships leading to regression analyses that took the small numbers into account. For the first of the hypotheses on the bivariate relationships among self- 
compassion, hyper-arousal and age of onset diagnosis of breast cancer, we examined whether greater self-compassion was related significantly to lower levels of arousal and a later onset of breast cancer. Results revealed meaningful relationships between the dependent variable, Self-compassion, and first, Hyperarousal $(r=-.49, p=.020)$ and second, Age diagnosed $(r=.60, p=.004)$.

\section{Examining the Hypotheses: Multiple Regressions}

We considered whether we could expect levels of self-compassion to predict lower hyper-arousal and, separately, later age of diagnosis (onset). Two separate regressions showed, as expected, that greater self-compassion predicted later onset of cancer $\left[F(1,19)=10.83, p=.004,\left(\beta=.60, p=.004, s r^{2}=.36 \%\right.\right.$; greater self-compassion also predicted lower hyper-arousal $[F(1,20)=6.37, p=.020,(\beta$ $\left.=-.49, p=.020, s r^{2}=.24 \%\right)$. Please see Table 1 for summary of two separate regression analyses for self-compassion.

Multiple regression analyses also demonstrated the reverse situation: that the variables Hyper-arousal and Age diagnosed with Breast Cancer were associated with levels of self-compassion. Hyper-arousal and Age of onset together significantly accounted for $49.0 \%$ of the variance in Self-Compassion $F(2,18)=10.61$, $p=.001$. Both Age diagnosed with breast cancer $\left(\beta=.55, p=.003, s r^{2}=.30 \%\right)$ and Hyper-arousal $\left(\beta=-.43, p=.017, s r^{2}=.18 \%\right)$ were significant unique predictors of, that is, closely associated with levels of Self-Compassion in individuals diagnosed with Breast Cancer. Please see Table 2 for summary of multiple regression analysis for self-compassion.

Table 1. Summary of regression analysis. Predictor variable for hyper-arousal and age of breast cancer onset-self-compassion.

\begin{tabular}{|c|c|c|c|c|}
\hline Variable & & $B$ & $S E B$ & $\beta$ \\
\hline \multicolumn{5}{|l|}{ Hyper-arousal } \\
\hline Self-Compassion & & -.51 & .20 & $-.49^{*}$ \\
\hline Total R2 & $.24^{*}$ & & & \\
\hline \multicolumn{5}{|c|}{ Age of Breast Cancer Onset } \\
\hline Self-Compassion & & .35 & .11 & $.60^{\star *}$ \\
\hline Total R2 & $.36^{* *}$ & & & \\
\hline
\end{tabular}

${ }^{\star} p<.05,{ }^{* *} p<.01,{ }^{* * *} p<.001$.

Table 2. Summary of the multiple regression analysis for self-compassion-predictor variables hyper-arousal and age of breast cancer onset.

\begin{tabular}{ccccc}
\hline Variable & & $B$ & SEB & $\beta$ \\
\hline Self-Compassion & & & & \\
Hyper-arousal &. & -.41 & .16 & $-.43^{\star}$ \\
Age of Breast Cancer Onset & & .95 & .28 & $.55^{* *}$ \\
Total R2 & $.54^{* * *}$ & & & \\
\hline
\end{tabular}

${ }^{\star} p<.05,{ }^{* *} p<.01,{ }^{* * *} p<.001$. 


\section{Discussion}

The results supported the hypotheses connecting Self-Compassion, hyperarousal and age of onset of diagnosis of breast cancer, and demonstrated that individuals who reported greater Self-Compassion levels experienced lower Hyperarousal and a later onset of Breast Cancer. Conversely individuals who reported less Self-Compassion also reported higher levels of Hyper-arousal and an earlier onset of breast cancer.

Therefore, we concluded from this study that attitudes involving self-compassion appeared to be associated strongly with the onset and experience of breast cancer. It seems that Self-Compassion may be an effective (protective) strategy for reducing negative physiological arousal (and its related impact on the immune system) and thus help to delay the onset of breast cancer.

\subsection{Implications of the Results}

The strong relationship between self-compassion, hyper-arousal and onset of breast cancer suggests that self-compassion is a powerful strategy for managing an individual's emotions, reducing negative physiological arousal and delaying the onset of breast cancer. This suggests that individuals who are likely to experience breast cancer as suggested by family and personal history, may delay the onset of this disease by developing strategies high in self-compassion. The under-utilization of effective emotion regulation strategies (such as being open to one's experiences without over-identifying, suppressing or avoiding, through the use of compassionate awareness); may reduce the capacity of an individual to self-sooth, thus heightening states of arousal and negatively impacting on immune system functioning.

Breast cancer participants in our study who engaged in self-compassion strategies were more likely to be experiencing lower negative arousal and to have experienced a later onset of breast cancer. The utilization of self-compassion as a strategy to calm emotions and reduce arousal, may have implications for individuals with a predisposition to breast cancer in that this strategy may provide opportunities to return the body to homeostasis more rapidly than for those who are less compassionate to themselves. Individual differences in the ability to effectively use self-compassion to manage emotions appear to be related to an individual's capacity to reduce negative physiological arousal and the subsequent immune response. Self-compassion may therefore through the reduction of elevated negative arousal (whether experienced as a result of exposure to adversity or intrinsic negative ways of relating to one-self) be an effective strategy for delaying onset of breast cancer.

To re-iterate, people predisposed to the development of breast cancer such as those with a family history of breast cancer may as a result of engaging in selfcompassion strategies, reduce one of the contributing physiological aspects of immune dysregulation (hyper-arousal) and subsequently may also delay any likely onset of the cancer. 


\subsection{Health and Family and Financial Implications}

Engaging in effective emotion regulation strategies such as self-compassion may not only provide personal benefits to the individual but financial benefits to the health system. A delay in onset may lessen the burden on families as children would be older and therefore the disease would be less likely to impact on the responsibilities associated with caring for a young family, managing a career and general life activities associated with these factors. The benefits of engaging in self-compassionate emotion regulation strategies would provide advantages for the individual, the family and the general community. Psychologists and health counsellors would screen their clients (with family histories of breast cancer) on their levels of self-compassion and physiological experiences (of hyper-arousal) and provide self-compassion strategies where required, to assist in the reduction of physiological arousal and the subsequent more effective immune response.

\section{Limitations of Study}

The recruitment of a larger sample of breast cancer participants for this research project was difficult due in part to breast cancer organisations' concerns about impacts on members' psychological experiences, including that involvement in the study could raise stress levels for a variety of reasons. In the circumstances 31 participants with breast cancer of whom 23 completed all data for the current study is consistent with other reported studies and we obtained valuable information as seen in the reported relationships.

Another limitation is that our study is correlational in nature and its use of self-reports including in regards to age of onset of cancer. We draw conclusions based on implications in the data, but further study is needed of an experimental kind to confirm that treatment outcomes from self-compassion training would lower hyper-arousal aspects. Also a limitation is the self-reporting of aspects related to age of onset of cancer diagnosis. As a reviewer of an earlier version of this paper suggested (and with which we concur), it would be preferable to develop a large-scale extensive (longitudinal) study of people before any illnesses are developed, with inbuilt controls where possible of the many variables that could influence age at which cancer is first diagnosed. In this way we could more easily identify factors that contribute. These large scale and/or longitudinal studies were beyond our scope in the current studies and hopefully others may be able to develop plans along these lines. However, our study despite the limitations has indicated that relationships exist between self-compassions and levels of stress (hyper-arousal) and between self-compassion and recollected age of onset of the disease. We recommend that the likely benefit of higher levels of self-compassion at least be recognised, and that help in learning such strategies be made available to those who might benefit from the approach.

\section{Acknowledgements}

It is clear we are dealing with a highly sensitive area; however, further studies are needed if we are to learn new information that can alleviate the pain and suffer- 
ing of many. We are grateful to the two Australian organisations who went out of their way to support our research, as we are also to those individuals with cancer from among our university students and their families who opted to be part of the study; we applaud their courage in fighting the disease and thank all those who participated in this study; and encourage them to be and to continue to be self-compassionate.

\section{References}

Australian Institute of Health and Welfare \& Australasian Association of Cancer Registries (2012). Cancer in Australia: An Overview, 2012. Cancer Series No. 74. Cat. no. CAN 70. Canberra: AIHW. http://www.aihw.gov.au/publication-detail/?id=60129542359

Brown, M. J., Thacker, L. R., \& Cohen, S. A. (2013). Association between Adverse Childhood Experiences and Diagnosis of Cancer. PLoS ONE, 8, e65524.

https://doi.org/10.1371/journal.pone.0065524

Every, G. S., \& Lating, J. M. (2002). The Link from Stress Arousal to Disease: In D. A. Meichenbaum (Ed.), Clinical Guide to Treatment of the Human Stress Response (2 ${ }^{\text {nd }}$ ed.). New York: Springer.

Gilbert, P. (2002). Evolutionary Approaches to Psychopathology and Cognitive Therapy. Journal of Cognitive Psychotherapy: An International Quarterly, 16, 263-294.

https://doi.org/10.1891/jcop.16.3.263.52515

Gilbert, P. (2007). Psychotherapy and Counselling for Depression (3rd ed.). London: Sage. https://doi.org/10.4135/9781446279830

Habersack, M., \& Luschin, G. (2013). Insecurities of Women Regarding Breast Cancer Research: A Qualitative Study. PloS oNe, 8, e81770. https://doi.org/10.1371/journal.pone.0081770

Hammad, M., Barsky, A., \& Regestein, Q. (2001). Correlation between Somatic Sensation Inventory Scores and Hyper-Arousal Scale Scores. Psychosomatics, 42, 29-34. https://doi.org/10.1176/appi.psy.42.1.29

Kanani, R., Davies, E. A., Hanchett, N., \& Jack, R. H. (2016). The Association of Mood Disorders with Breast Cáncer Survival: An Investigation of Linked Cáncer Registration and Hospital Administration Data for South East England. Psycho-Oncology, 25, 19-27. https://doi.org/10.1002/pon.4037

Kearney, K. G., \& Hicks, R. E. (2016). Early Nurturing Experiences, Self-Compassion, Hyperarousal and Scleroderma. The Way We Relate to Ourselves May Determine Disease Progression. International Journal of Psychological Studies, 8, 16-25. https://doi.org/10.5539/ijps.v8n4p16

Neff, K. (2003a). Self-Compassion: An Alternative Conceptualization of a Healthy Attitude toward Oneself. Self and Identity, 2, 85-101. https://doi.org/10.1080/15298860309032

Neff, K. D. (2003b). The Development and Validation of a Scale to Measure Self-Compassion. Self and Identity, 2, 223-250. https://doi.org/10.1080/15298860309027

Neff, K. D., Kilpatrick, K. L., \& Rude, S. S. (2007). Self-Compassion and Adaptive Psychological Functioning. Journal of Research in Personality, 41, 139-154.

https://doi.org/10.1016/j.jrp.2006.03.004

Pace, T., Negi, L. T., Ademe, D., Cole, S., Sivilli, T., Brown, T., Raison, C. L. et al. (2009). Effect of Compassion Meditation on Neuroendocrine, Innate Immune and Behavioural 
Responses to Psychosocial Stress. Psychoneuroendocrinology, 34, 87-98. https://doi.org/10.1016/j.psyneuen.2008.08.011

Palesh, O., Butler, L. D., Koopman, C., Giese-Davis, J., Carlson, R., \& Spiegel, D. (2007). Stress History and Breast Cancer Recurrence. Journal of Psychosomatic Research, 63, 233-239. https://doi.org/10.1016/j.jpsychores.2007.05.012

Przezdziecki, A., Sherman, K. A., Baillie, A., Taylor, A., Foley, E., \& Stalgis-Bilinski, K. (2013). My Changed Body: Breast Cancer, Body Image, Distress and Self-Compassion. Psycho-Oncology, 22, 1872-1879. https://doi.org/10.1002/pon.3230

Rice, P. L. (1999). Stress and Health (2nd ed.). California: Brookes/Cole.

Schore, A. N. (1994). Affect Regulation and the Origin of the Self: The Neurobiology of Emotional Development. Mahwah, NJ: Erlbaum.

Selye, H. (1976). Forty Years of Stress Research: Principal Remaining Problems and Conceptions. Canadian Medical Association Journal, 115, 53-56.

Sephton, S., \& Spiegel, D. (2003). Circadian Disruption in Cancer: A Neuroendocrine-Immune Pathway from Stress to Disease. Brain, Behaviour and Immunity, 17, 321-328. https://doi.org/10.1016/S0889-1591(03)00078-3

Sharp, L., O’Leary, E., Kinnear, H., Gavin, A., \& Drummond, F. (2015). Cancer-Related Symptoms Predict Psychological Wellbeing among Prostate Cancer Survivors: Results from the PiCTure Study. Psycho-Oncology, 25, 282-291.

https://doi.org/10.1002/pon.3909

Sharpiro, S. L., Astin, J. A., Bishop, S. R., \& Cordova, M. (2005). Mindfulness-Based Stress Reduction for Health Care Professionals: Results from a Randomised Trial. International Journal of Stress Management, 12, 164-176.

https://doi.org/10.1037/1072-5245.12.2.164

Wren, A., Somers, T. J., Wright, M. A., Goetz, M. C., Leary, M. R., Fras, A. M., Keefe, F. J. et al. (2011). Self-Compassion in Patients with Persistent Musculoskeletal Pain: Relationship of Self-Compassion to Adjustment to Persistent Pain. Journal of Pain and Symptom Management, 43, 759-770.

https://doi.org/10.1016/j.jpainsymman.2011.04.014

Submit or recommend next manuscript to SCIRP and we will provide best service for you:

Accepting pre-submission inquiries through Email, Facebook, LinkedIn, Twitter, etc. A wide selection of journals (inclusive of 9 subjects, more than 200 journals)

Providing 24-hour high-quality service

User-friendly online submission system

Fair and swift peer-review system

Efficient typesetting and proofreading procedure

Display of the result of downloads and visits, as well as the number of cited articles

Maximum dissemination of your research work

Submit your manuscript at: http://papersubmission.scirp.org/

Or contact psych@scirp.org 